\title{
RESEARCH
}

Open Access

\section{Disruption of Midkine gene reduces traumatic brain injury through the modulation of neuroinflammation}

Seiya Takada', Harutoshi Sakakima ${ }^{1 *} \mathbb{D}$, Takahiro Matsuyama², Shotaro Otsuka' ${ }^{1}$ Kazuki Nakanishi', Kosuke Norimatsu', Yuki Itashiki ${ }^{1}$, Akira Tani ${ }^{1}$ and Kiyoshi Kikuchi ${ }^{3}$

\begin{abstract}
Background: Midkine (MK) is a multifunctional cytokine found upregulated in the brain in the presence of different disorders characterized by neuroinflammation, including neurodegenerative disorders and ischemia. The neuroinflammatory response to traumatic brain injury (TBI) represents a key secondary injury factor that can result in further neuronal injury. In the present study, we investigated the role of endogenous MK in secondary injury, including neuroinflammation, immune response, and neuronal apoptosis activity, after TBI.
\end{abstract}

Methods: Wild type $\left(\mathrm{Mdk}^{+/+}\right)$and MK gene deficient $\left(\mathrm{Mdk}^{-/}\right)$mice were subjected to fluid percussion injury for TBI models and compared at 3, 7, and 14 days after TBI, in terms of the following: brain tissue loss, neurological deficits, microglia response, astrocytosis, expression of proinflammatory M1 and anti-inflammatory M2 microglia/ macrophage phenotype markers, and apoptotic activity.

Results: As opposed to $\mathrm{Mdk}^{+/+}$mice, $\mathrm{Mdk}^{-/-}$mice reported a significantly reduced area of brain tissue loss and an improvement in their neurological deficits. The ratios of the lba1-immunoreactive microglia/macrophages in the perilesional site were significantly decreased in $\mathrm{Mdk}^{-/-}$than in the $\mathrm{Mdk}^{+/+}$mice at 3 days after TBI. However, the ratios of the glial fibrillary acidic protein immunoreactive area were similar between the two groups. The M1 phenotype marker (CD16/32) immunoreactive areas were significantly reduced in $\mathrm{Mdk}^{-/-}$than in the $\mathrm{Mdk}^{+/+}$mice. Likewise, the mRNA levels of the M1 phenotype markers (TNF-a, CD11b) were significantly decreased in $\mathrm{Mdk}^{-1-}$ mice than in $\mathrm{Mdk}^{+/+}$mice. Furthermore, flow cytometry analysis identified the $\mathrm{M} 2$ markers, i.e., $\mathrm{CD} 163^{+}$ macrophages cells and arginase- $1^{+}$microglia cells, to be significantly higher in $\mathrm{Mdk}^{-/-}$than in $\mathrm{Mdk}^{+/+}$mice. Finally, the ratios of apoptotic neurons were significantly decreased in the area surrounding the lesion in $\mathrm{Mdk}^{-/-}$than in $\mathrm{Mdk}^{+/+}$mice following TBl.

Conclusion: Our findings suggest that MK-deficiency reduced tissue infiltration of microglia/macrophages and altered their polarization status thereby reducing neuroinflammation, neuronal apoptosis, and tissue loss and improving neurological outcomes after TBI. Therefore, targeting MK to modulate neuroinflammation may represent a potential therapeutic strategy for TBI management.

Keywords: Midkine, Traumatic brain injury, Microglia/macrophages, M1/M2 phenotype, Neuroinflammation

\footnotetext{
* Correspondence: sakaki@health.nop.kagoshima-u.ac.jp

${ }^{1}$ Department of Physical Therapy, School of Health Sciences, Faculty of

Medicine, Kagoshima University, 8-35-1, Sakuragaoka, Kagoshima 890-8544,

Japan

Full list of author information is available at the end of the article
}

(C) The Author(s). 2020 Open Access This article is distributed under the terms of the Creative Commons Attribution 4.0 International License (http://creativecommons.org/licenses/by/4.0/), which permits unrestricted use, distribution, and reproduction in any medium, provided you give appropriate credit to the original author(s) and the source, provide a link to the Creative Commons license, and indicate if changes were made. The Creative Commons Public Domain Dedication waiver (http://creativecommons.org/publicdomain/zero/1.0/) applies to the data made available in this article, unless otherwise stated. 


\section{Background}

Traumatic brain injury (TBI) is one of the leading causes of death and disability nowadays. Indeed, more than half of trauma-related deaths can be attributed to TBI, and approximately $40 \%$ of TBI survivors in the USA develop a long-term disability [1, 2]. Its intricate pathophysiological process involves traumatic mechanical forces, which result in either primary or secondary brain injury in endothelium or the brain parenchyma [3, 4]. Secondary injury follows the primary injury-mediated neuroinflammation, immune response, oxidative stress, mitochondrial dysfunction, and apoptosis and may last from hours to days after the initial insults [5]. Furthermore, secondary injury hampers the extant neuroprotection and neurorepair mechanisms, leading to delayed and limited neurobehavioral recovery after TBI [6]. Typically, inflammation (i.e., the activation of resident microglia) is believed to play an important role in these secondary changes $[7,8]$.

Microglia cells are the primary mediators of the innate immune response in the central nervous system (CNS). Resident microglia cells are rapidly mobilized to the site of injury and can be polarized into either a detrimental (M1) or a beneficial (M2) phenotype depending on their host tissue microenvironments [9, 10]. M1- and M2-like microglia release several factors responsible for the modulation of secondary injury and recovery after TBI, including proinflammatory and anti-inflammatory cytokines, chemokines, nitric oxides, prostaglandins, growth factors, and superoxide species [7, 8, 11]. M1- and M2like microglia/macrophages work in concert to fine-tune the inflammatory responses, scavenge debris, and promote the remodeling and repair following TBI [8]. Notably, the proinflammatory cytokines released by M1-like microglia, such as tumor necrosis factor- $\alpha$ (TNF- $\alpha$ ) and interleukin-1 $\beta$ (IL-1 $\beta$ ), are believed to contribute to neuronal cell damage $[8,12,13]$. Indeed, inhibiting microglia activation-mediated inflammation, through the regulation of both the nuclear factor-kappa B (NF-kB) and the mitogen-activated protein kinase (MAPK) signal pathway, was suggested to improve the neurobehavioral function after TBI [14]. In addition, acute administration of atorvastatin, anti-inflammatory, and immunomodulatory agents was found to attenuate the M1 microglia/ macrophages phenotype and simultaneously increase the M2 phenotype post-TBI [5]. Therefore, the inhibition of microglia/macrophages activation could be a valuable therapeutic option for improving recovery in TBI patients.

Midkine $(\mathrm{MK})$ is a retinoic acid-inducible heparinbinding growth factor that is transiently expressed in the mid-gestational period of mouse embryogenesis and is downregulated to inconsiderable levels in healthy adults [15]. However, MK remains upregulated during tissue repair and in several pathological conditions, including malignancies and inflammatory diseases [16]. For example, MK is expressed in the senile plaques detected in the brain of Alzheimer's disease patients [17], in the areas surrounding cerebral infarcts [18], in injured spinal cords of rats [19], and in various human neoplasms [20]. MK promotes migration, proliferation, survival, growth, reproduction and repair, angiogenesis, and gene expression of target cells [15]. Furthermore, MK facilitates the migration of macrophages and neutrophils in various peripheral tissues, including kidney, vascular organs, and liver [21-24]. Recent studies have demonstrated that MK is involved in both the onset and progression of autoimmune diseases such as multiple sclerosis and rheumatoid arthritis [25-27]. Similarly, MK aggravates experimental autoimmune encephalomyelitis (EAE) by suppressing the development of tolerogenic dendritic cells (DCregs), thereby impairing the differentiation of regulatory $\mathrm{T}$ cells (Treg) [28]. Therefore, MK inhibition using RNA aptamers may provide an effective therapeutic strategy against autoimmune diseases [26]. With regard to the CNS, MK was found to modulate neuroinflammation in both the cortex and the striatum as a result of lipopolysaccharide (LPS) and amphetamine injections $[29,30]$. However, the role of MK in TBI-induced neuroinflammation is yet to be elucidated. Considering that $\mathrm{MK}$ may regulate $\mathrm{TBI}$-induced neuroinflammation, it represents a potential therapeutic option for TBI management in patients. Our previous studies demonstrated the essential role of endogenous $\mathrm{MK}$ in the growth, survival, and migration of various cells using wild type $\left(\mathrm{Mdk}^{+/+}\right)$and $\mathrm{MK}$ gene-deficient $\left(\mathrm{Mdk}^{-/-}\right)$mice $[31,32]$. Here, $\mathrm{Mdk}^{+/+}$and $\mathrm{Mdk}^{-/-}$ mouse models were utilized to investigate the role of endogenous $\mathrm{MK}$ in secondary injury following TBI, which includes neuroinflammation, immune response, M1 and M2 microglia/macrophage phenotype polarization, and apoptosis activity.

\section{Methods}

\section{Animals}

In the present study, a total of $64 \mathrm{Mdk}^{+/+}$and $\mathrm{Mdk}^{-/-}$ mice C57BL/6 mice (8-32 week of age; $22.3 \pm 2.4 \mathrm{~g}$ of weight) were generated as previously described [31, 32]. In an attempt to reduce the number of animal sacrificed in the current research, both male and female mice were used. Mice were bred with a 12-h light/dark cycle and maintained at a controlled temperature $\left(22.0 \pm 1.0^{\circ} \mathrm{C}\right)$, with free access to food and water. The experimental protocol was approved by the ethics board of the Institute of Experimental Animal Science of Kagoshima University. 


\section{Fluid percussion injury model for TBI}

Fluid percussion injury (FPI) was adopted for TBI preparation. Briefly, mice were anesthetized with 1.5-2.0\% isoflurane (Pfizer Inc., Tokyo, Japan) using the MKA110 small animal anesthetizer (Muromachi kikai, Tokyo, Japan). Mice were fixed in a stereotaxic frame, and the hair on their head was removed and sterilized with 70\% ethanol. Their skull was then exposed through a midline scalp incision, and craniotomy was performed over the right parietal bone. In addition, a circular window (3.0 mm of diameter) was drilled using a dental drill to prevent damage to the dura. The surgical site was selected at $3.0 \mathrm{~mm}$ posterior and $3.0 \mathrm{~mm}$ on the right of the Bregma. Moreover, a male luer lock $(3.0 \mathrm{~mm}$, Isis, Osaka, Japan) filled with saline was firmly fixed on the surgical site using dental cement (Provista, Morita, Osaka, Japan). Successively, a tube of the FPI device (Dragonfly R \& D INC. Model HPD-1700, USA) was tightly attached to a three-way stopcock that connected the male luer lock hub. Therefore, mice were subjected to experimental FPI, at a water pressure impact of 2.0$3.0 \mathrm{~atm}$. After injury, we confirmed hemorrhage and brain edema in the circular window of the skull. Mice that present cessation of breathing or appendicular convulsions were included in this study as TBI models [33]. Finally, mice were left to recover in their cages with sufficient food and water. The weight of each mouse was periodically recorded after TBI.

\section{Histological and immunohistochemical analyses}

Mice were sacrificed at 3, 7, and 14 days after injury for histological and immunohistochemical analyses $(n=5 /$ group at each time-point). Deeply anesthetized with sodium pentobarbital, mice were perfused through the heart with physiological saline, followed by $4 \%$ paraformaldehyde in a $0.1 \mathrm{M}$ phosphate buffer (pH 7.4). Subsequently, their brain was carefully removed and cut into two tissue blocks (2-mm anterior to $2-\mathrm{mm}$ posterior to the injury site) using a brain slicer. The sections were then immersed in $4 \%$ paraformaldehyde in a $0.1 \mathrm{M}$ phosphate buffer $(\mathrm{pH} 7.4)$ at $4{ }^{\circ} \mathrm{C}$ overnight. After fixation, the tissue was processed for histology and immunohistochemistry analyses. The paraffin-embedded coronal brain sections $(4 \mu \mathrm{m}$ thick) were stained with hematoxylin and eosin (HE); the HE sections of the center of injury site (Fig. 1a) were imaged at $\times 4$ magnification using a microscope (Olympus Corp, DP21, Tokyo, Japan). The brain tissue loss at 7 and 14 days was analyzed using the Scion Image Software 4.0.3 (Scion Corp, Frederick, MD, USA), considering that the brain tissue was completely missing after TBI.

The coronal sections were immunostained with the following antibodies: rabbit anti-glial fibrillary acidic protein (GFAP; a marker of activated astrocytes) (Cosmo
Bio Co., Japan; RO1003), rabbit anti-ionized calciumbinding adaptor molecule1 (Iba1; a marker of resting microglia/macrophage) (Wako, Osaka, Japan; 01919741), rat anti-CD16/32 (a marker of M1 microglia/ macrophage) (BD Bioscience, US; \#553142), rabbit antiarginase-1 (a marker of M2 microglia/macrophage) (Cell Signaling Technology, Inc., US; \#93668), rabbit antiactivated caspase-3 (a marker of apoptotic activity) (Proteintech Group, Inc.; USA; 19677-1-AP), and mouse anti-neuronal nuclei (NeuN; a marker of neuron) (Abcam plc, Cambridge, UK; ab104224).

Following deparaffinization and rehydration, the endogenous peroxidase was blocked with methanol containing 3.0\% hydrogen peroxide for $10 \mathrm{~min}$. The sections were then rinsed three times $(5 \mathrm{~min}$ each) with phosphate-buffered saline (PBS, pH 7.6) and blocked with $10 \%$ skimmed milk in PBS for $20 \mathrm{~min}$. Successively, all sections were individually incubated at $4{ }^{\circ} \mathrm{C}$ overnight with the following antibodies: rabbit anti-GFAP (1:3000), rabbit anti-Iba1 (1:1000), rat anti-CD16/32 (1:500), rabbit anti-arginase-1 (1:1000), and rabbit anti-caspase-3 (1:200). The sections were then washed in PBS three times for $5 \mathrm{~min}$ each and incubated for $60 \mathrm{~min}$ with goat anti-rabbit IgG conjugated to either a peroxidase-labeled dextran polymer (EnVision; Dako, CA, USA) or biotinylated anti-rat IgG (1:200) and then stained with $A B C$ (ABC Kit: Vector Laboratories, Burlingame, CA, USA), according to the manufacturer's instructions. Finally, the sections were rinsed with PBS and their immunoreactivity was visualized through diaminobenzidine staining.

The co-localization of the rabbit anti-caspase-3 (1: $200)$ and the mouse anti-NeuN (1:500) immunoreactivities were examined through immunofluorescence staining. After incubation with two primary antibodies and PBS wash, the sections were incubated for $60 \mathrm{~min}$ with both the FITC-conjugated goat anti-rabbit IgG (1: 200) and Alexa Fluor 555-conjugated goat anti-mouse IgG antibodies (1:200). The sections were washed with PBS and counterstained with 4', 6-diamino-2-phenylindole for $10 \mathrm{~min}$. Finally, they were mounted with an aqueous mounting media and immunofluorescent staining was observed with a fluorescence microscope (EVOS f1; AMG, Mill Creek, WA, USA).

\section{Evaluation of neurological deficits}

Mice ( $n=5$ /group) were scored neurologically for focal deficits using a 28-point neurological severity score (NSS) [34]. Briefly, this system included comprehensive assessment of motor, sensory, reflex, and balance. The highest test score possible was 28 points, representing the most serious neurological impairment. Prior to TBI, all mice scored zero. Following TBI, the NSS was administered to mice at 3,7 , and 14 days, and the scoring was performed by two individuals. 

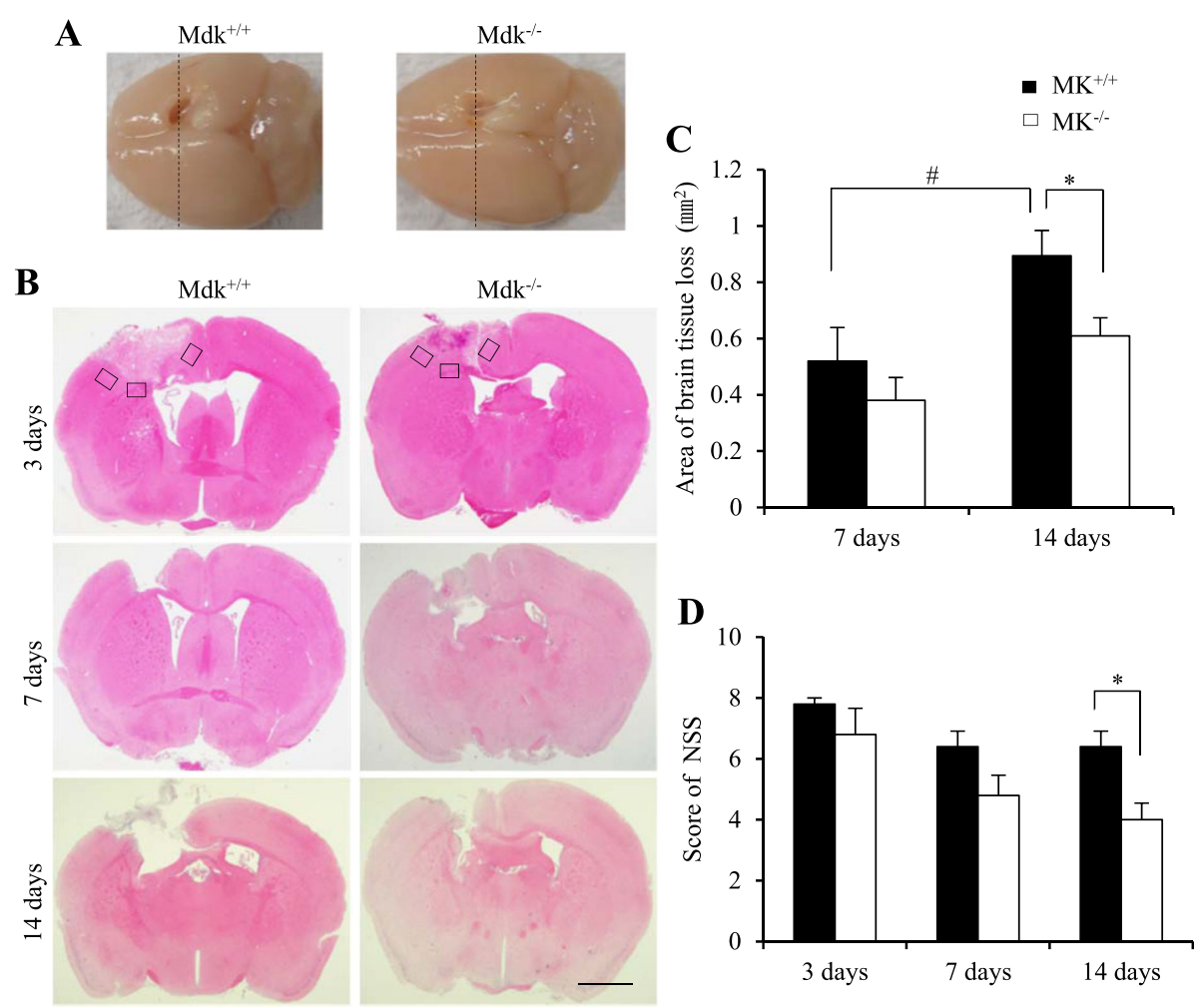

Fig. 1 Effect of MK-deficiency on brain tissue loss and recovery of neurological deficits. Tissue loss after TBI detected using coronal hematoxylin and eosin (HE) staining, including a section obtained from the center (dashed line) of the injury site and the macro brain photographed at 14 days (a). TBI led to an obvious tissue loss at 7 and 14 days. Three rectangular areas in all mice were used for the quantitative analysis of the immunolabeled areas at each time-point (b). Mdk $^{-/-}$mice presented a significantly reduced tissue loss than the Mdk ${ }^{+/+}$mice at 14 days after TBI (c). The neurological deficits in all $\mathrm{Mdk}^{-/-}$mice were significantly ameliorated at 14 days after TBI (d). Data are presented as mean \pm SE $(n=5$ mice/group). ${ }^{*} p<0.05$ (comparison with $\mathrm{MK}^{+/+}$and $\mathrm{Mdk}^{-/-}$). $\# p<0.05$ (comparison with 7 days and 14 days). Scale bar $=1 \mathrm{~mm}$ (all panels). TBI, traumatic brain injury

\section{Quantitative analysis of immunolabeled areas}

Three areas surrounding the lesion in each immunostained section were imaged at $\times 40$ magnification using a microscope and a camera without visual field overlap (Fig. 1a). The ratios of GFAP-, Iba1-, CD16/32-, ariginase-1-, and caspase-3-positive cells were quantitatively measured between the two groups in coronal sections using the Scion Image software 4.0.3 at 3, 7, and 14 days after TBI. Moreover, at 7 days after TBI, sections obtained from two areas in the motor cortex of the surrounding lesion, with a co-localization of caspase- 3 and NeuN were imaged $(\times 20$ magnification $)$ following immunofluorencent staining. Furthermore, the ratios of caspase-3- and NeuN-positive neurons were counted in the surrounding lesion $\left(0.21 \mathrm{~mm}^{2}\right)$ and a quantitative analysis of each immunolabeled area was performed by two to three individuals.

\section{Reverse transcriptase quantitative PCR (RT-qPCR)}

All mice were euthanized through deep anesthesia with sodium pentobarbital 3 days after TBI ( $n=3-4$ /group). Therefore, the total RNA was isolated from the injured brain $(100 \mathrm{mg})$ using the Trizol reagent (Cosmo Bio Co., Japan, 5631) and the cDNA was synthesized using the Verso cDNA Kit (Thermo Fisher Scientific, Massachusetts, USA) according to the manufacturer's instructions. This was then amplified by reverse transcriptase PCR using the SYBR green PCR Master Mix (Applied Biosystem; Thermo Fisher Scientific, Inc.). Specifically, a twostep qPCR was performed $\left(95^{\circ} \mathrm{C}\right.$ for $15 \mathrm{~s}, 60^{\circ} \mathrm{C}$ for $30 \mathrm{~s}$ for 40 cycles) with specific primers. The comparative cycle threshold $(\mathrm{Ct})$ analysis $(\Delta \Delta \mathrm{Ct})$ was performed to evaluate the fold-change in mRNA expression using GAPDH expression as the reference. We checked that GAPDH expression does not differ between experiment groups. The sequences of the primer pairs for the M1 phenotype genes are the following: (1) tumor necrotic factor- $\alpha$ (TNF- $\alpha)$ : forward, 5'-CGTCGTAGCAAACC ACCAAAG-3' and reverse, 5'-GAGATAGCAAATCG GCTGACG-3'; (2) CD16: forward, 5'-TTTGGACACC CAGATGTTTCA-3' and reverse, 5'-GTCTTCCTTG AGCACCTGGATC-3'; (3) CD11b: forward, 5'CCAAGACGATCTCAGCATCA-3' and reverse, 5' TTCTGGCTTGCTGAATCCTT-3'. In contrast, the 
sequences of the primer pairs for the M2 phenotype genes are as follows: (1) arginase-1: forward, 5' TCACCTGAGCTTTGATGTCG-3' and reverse, 5' CTGAAAGGAGCCCTGTCTTG-3'; (2) CD206; forward, 5'-CAAGGAAGGTTGGCATTTGT-3' and reverse, $\quad 5^{\prime}$-CCTTTCAGTCCTTTGCAAGC-3'. The sequences of the primer pairs for the GAPDH genes are as follows: forward, 5'-AACTTTGGCATTGT GGAAGG-3' and reverse, 5'-ACACATTGGGGTAG GAACA-3'.

\section{Flow cytometry}

Deeply anesthetized with sodium pentobarbital, mice were perfused with physiological saline through the heart and their ipsilateral brain was promptly removed 3 days after TBI ( $n=4 /$ group). Tissue debris were then incubated via Percoll gradient centrifugation to obtain a single-cell suspension. Prior to antibody labeling, the cell suspension was incubated at $4{ }^{\circ} \mathrm{C}$ for $20 \mathrm{~min}$ with the anti-mouse CD16/32 FACS buffer (BD pharmingen, New Jersey, USA; Clone 2.4G2) in FACS buffer (HBSS buffer containing $2 \%$ FBS) to prevent non-specific binding of immunoglobulin to macrophage Fc receptors. Cells were lysed in the FACS buffer containing the following fluorescent labels and incubated at $4{ }^{\circ} \mathrm{C}$ for 20 min in the dark: CD4- BV421(BD pharmingen, NJ, USA; Clone GK1.5), CD3e- BV510 (Biolegend, CA, USA; Clone 145-2C11), CD45.2- Alexa Fluor 700 (Biolegend, CA, USA; Clone 104), CD25- PECy7 (Biolegend; Clone PC61), and CD11b-FITC (TONBO bioscience, CA, USA; Clone M1/70). To differentiate M1 and M2 microglia/ macrophages phenotype, we used antibodies against the iNOS-PE (eBioscience, California, USA, 12-5920-82; Clone CXNFT), arginase-1-APC (eBioscience, CA, USA, 17-3697-82; Clone A1exF5), CD80-PE (eBioscience, CA, USA, 12-0801-81; Clone 16-10A1), and CD163-APC (eBioscience, CA, USA, 17-1631-82; Clone TNKUPJ) monoclonal antibodies. All cells were then incubated at $4{ }^{\circ} \mathrm{C}$ for $20 \mathrm{~min}$ in the dark, and their fluorescence was analyzed with the FAC Scan analyzer (BD bioscience, CA, USA). Finally, we calculated the percentages of total microglia or macrophage cells number.

\section{Statistical analysis}

Statistical analyses were performed with either parametric or non-parametric tests after Shapiro Wilk test. Either the independent Student $t$ test or the MannWhitney $U$ test was applied for between-group analyses. The time course for NNS was analyzed through the Friedman test. In addition, the time course for the percentages of immunostained areas was analyzed using either a one-way analysis of variance (ANOVA) or the Kruskal-Wallis test, followed by Bonferroni's post hoc tests for multiple comparisons. A $p$ value of $<0.05$ was considered statistically significant. Data are expressed as mean \pm standard error (SE). All data were analyzed using SPSS version 24 (IBM, Chicago, IL, USA).

\section{Results \\ MK-deficiency alleviates brain tissue loss and neurological deficits following TBI}

To assess the FPI-induced morphological changes, HEstained sections of the lesions were evaluated at 3,7 , and 14 days after TBI (Fig. 1a, b). Substantial brain damage was seen in the cortical layers with an extension into the corpus callosum of the ipsilateral hemisphere at 3 days after injury in all mice. Considering that brain tissue loss became obvious at 7 and 14 days following TBI, we estimated the area of brain tissue loss in all mice at these two time-points. While $\mathrm{Mdk}^{+/+}$mice presented a significant increase in the area of brain tissue loss over time (Fig. 1c; $p=0.037, t$ test), the same was not observed for $\mathrm{Mdk}^{-/-}$mice. In contrast, at 14 days after TBI, $\mathrm{Mdk}^{-/-}$ mice showed a significantly smaller area of brain tissue loss compared to $\mathrm{Mdk}^{+/+}$mice (Fig. 1c; $p=0.033, t$ test). Nonetheless, a significant difference between $\mathrm{Mdk}^{+/+}$ and the $\mathrm{Mdk}^{-/-}$mice at 7 days after TBI was not observed regarding brain tissue loss.

The neurological deficits in mice were assessed using the NSS system (Fig. 1d). While they were found to be absent in both mice groups before TBI, all mice exhibited severe neurological deficits after the injury, which however improved with time. Specifically, as opposed to $\mathrm{Mdk}^{+/+}$mice, the neurological deficits in $\mathrm{Mdk}^{-/-}$mice were significantly ameliorated at 14 days after TBI $(p=$ 0.032, Mann-Whitney $U$ test).

\section{MK-deficiency lessened neuroinflammation through a decrease in microglia responses and in M1-like microglia/ macrophages expression during the early phase after TBI} To investigate the inflammatory responses to TBI, microglial or astrocytic responses were determined via immunohistochemistry (Fig. 2). We found significantly lesser Iba1-immunoreactive microglia/macrophages at the perilesional site of $\mathrm{Mdk}^{-/-}$than those of $\mathrm{Mdk}^{+/+}$ mice at 3 days $(p=0.0001$, Mann-Whitney $U$ test). In addition, the density and processes of Iba1-positive microglia cells in $\mathrm{Mdk}^{-1-}$ mice were smaller than those in $\mathrm{Mdk}^{+/+}$mice (Fig. 2a). Although Iba1-positive cells slightly increased at 7 days, no significant difference in the time course after TBI was observed between the groups. Moreover, Iba1-immunoreactivities in $\mathrm{Mdk}^{+/+}$ mice significantly decreased at 14 days than at 3 days after TBI ( $p=0.001$, one-way ANOVA) and exhibited similar degrees of Iba1-immunoreactive areas to $\mathrm{Mdk}^{-1-}$ mice at 14 days after TBI. Although GFAP-positive astrocyte immunoreactivity was observed at 3 days in all mice, it significantly reduced at 14 days than at 3 days 


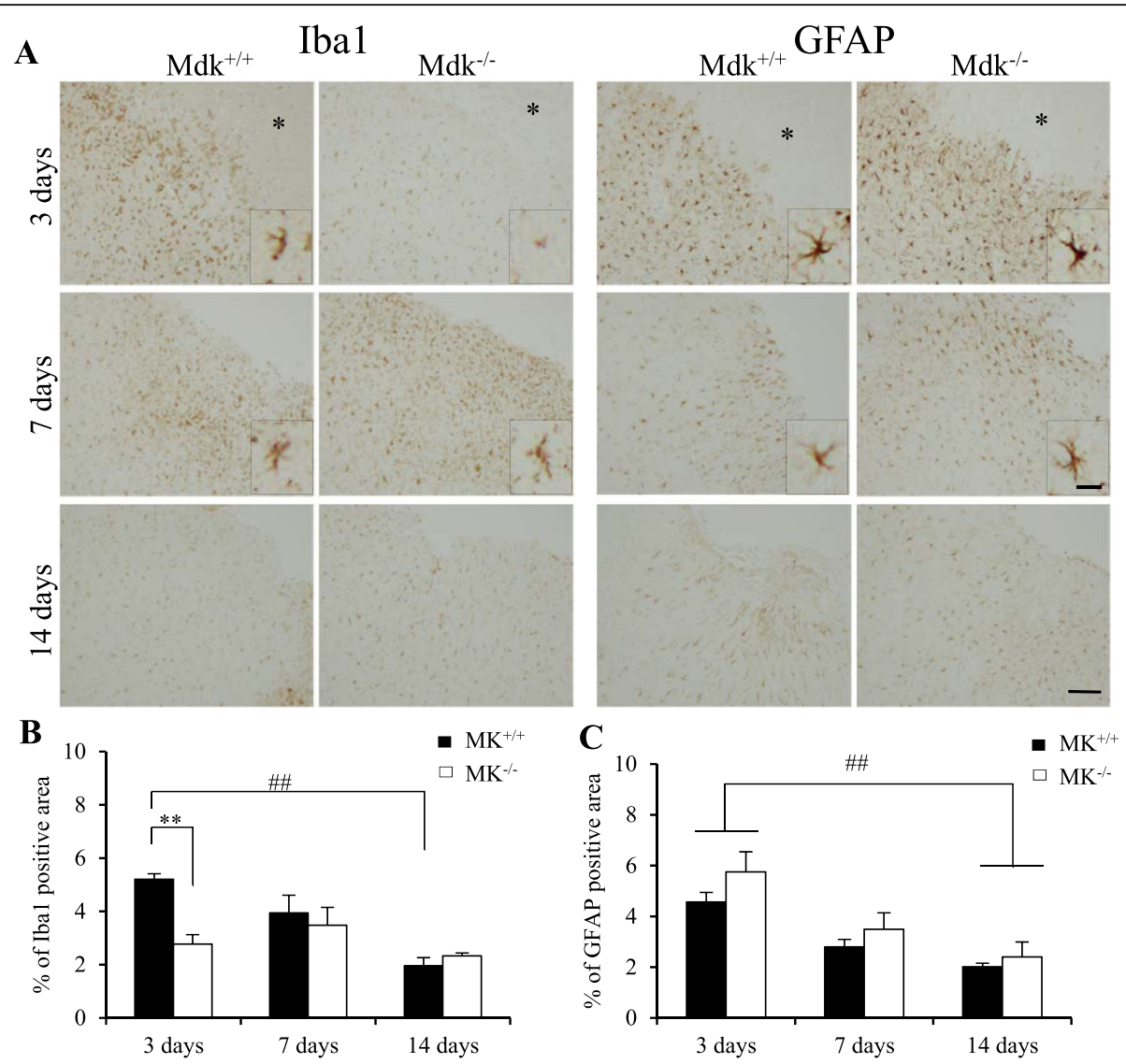

Fig. 2 Effect of MK-deficiency on microglia response and astrocytosis after TBI. Iba1-immunoreactive microglia/macrophages surrounding the lesion site $\left(^{*}\right)$ was significantly decreased in $\mathrm{Mdk}^{-/-}$compared to $\mathrm{Mdk}^{+/+}$mice at 3 days after TBI $(\mathbf{a}, \mathbf{b})$. However, there were no difference in GFAP-immunoreactivities between $\mathrm{MK}^{+/+}$and $\mathrm{Mdk}^{-/-}$mice at each time-point. (a, c). Furthermore, although the density and processes of Iba1positive microglia cells in $\mathrm{Mdk}^{-/-}$mice were smaller than those of $\mathrm{Mdk}^{+/+}$mice at 3 days, GFAP-positive astrocytes were not observed (a). Data are presented as mean $\pm \mathrm{SE}\left(n=5\right.$ mice/group). ${ }^{* *} p<0.01$ (comparison with $\mathrm{MK}^{+/+}$and $\mathrm{Mdk}^{-/-}$). \#\#p $<0.01$ (comparison with 3 days and 14 days). Scale bar $=100 \mu \mathrm{m}$ (all panels) and $20 \mu \mathrm{m}$ (all high magnification panels). GFAP, glia fibrillary acidic protein

after TBI $(p<0.05$, one-way ANOVA). However, no significant difference was observed in the ratios of GFAPpositive over GFAP-negative areas between mice at any time-point after TBI (Fig. 2c). Unexpectedly, morphological changes between mice such as density and processes of GFAP-positive astrocytes were not observed (Fig. 2a).

Successively, considering that Iba1-positive microglia/ macrophages cells were significantly decreased in acute phase after TBI in $\mathrm{Mdk}^{-/-}$mice, the expression of microglia/macrophages was determined through immunohistochemistry using M1 and M2 phenotypes markers at 3 and 7 days (Fig. 3a, b). The M1 and M2 phenotype markers (CD16/32 and arginase-1, respectively) were expressed in the perilesional site in all mice at 3 days; however, they significantly decreased at 7 days after TBI (Fig. 3a, b; $p<0.01, t$ test). Although the ratios of the CD16/32-immunoreactive area were significantly reduced in $\mathrm{Mdk}^{-/-}$than in $\mathrm{Mdk}^{+/+}$mice at 3 days (Fig. 3b; $p=0.006, t$ test,), a significant difference between mice at 7 days after TBI was not observed. Similarly, at each time-point after TBI, the ratios of the arginase-1immunoreactive area were not significantly different between mice at any time of analysis (Fig. 3b).

\section{MK-deficiency altered the polarization of M1 and M2 microglia/macrophages phenotype markers}

Polarized microglia/macrophages can be distinguished by surface marker expression and cytokine/chemokine production. Therefore, we examined the markers and the functional cytokine mRNA levels of the M1 (TNF- $\alpha$, CD16, CD11b) and M2 (arginase-1, CD206) phenotypes by RT-qPCR analysis at 3 days (Fig. 3c). The mRNA levels of the M1 phenotype markers (TNF- $\alpha$, CD11b) were significantly lower in $\mathrm{Mdk}^{-/-}$than in $\mathrm{Mdk}^{+/+}$mice $(p<0.05, t$ test). In contrast, the mRNA levels of the M2 phenotype markers were similar between mice.

In addition, cells isolated from the brain lesions were analyzed for $\mathrm{CD}^{+}{ }^{+} \mathrm{CD} 25^{+} \mathrm{T}$ cell (Treg), M1 phenotype markers $\left(\mathrm{CD}^{+} 0^{+}, \mathrm{iNOS}^{+}\right)$, and $\mathrm{M} 2$ phenotype markers 

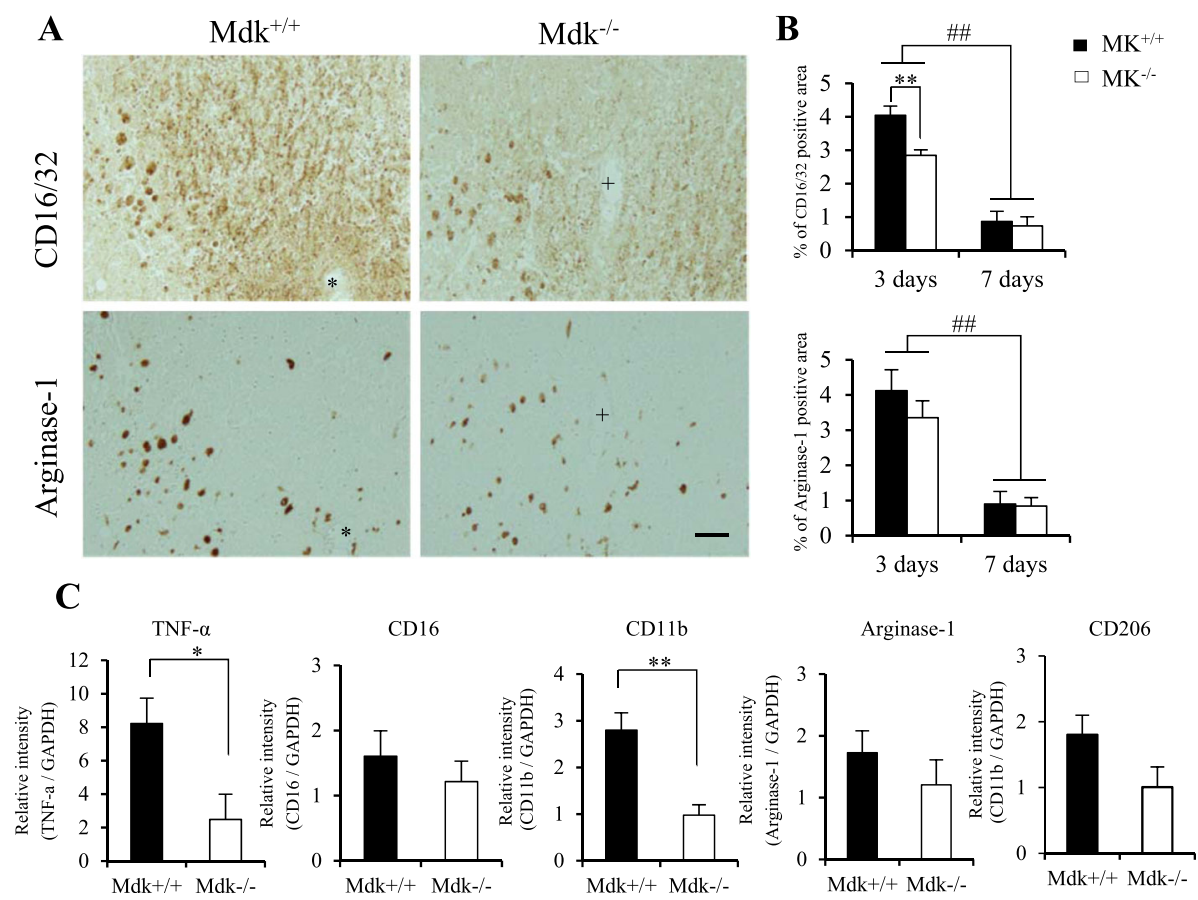

Fig. 3 Effect of MK-deficiency on M1 and M2 microglia/macrophages phenotype marker after TBI. The M1 and M2 phenotype markers (CD16/32 and arginase-1, respectively) were expressed in the perilesional site of $\mathrm{Mdk}^{+/+}$and $\mathrm{Mdk}^{-/-}$mice at 3 days (a). The immunohistochemical staining was performed through serial sections of mice (corresponding to * and + in a). The ratios of the CD16/32-immunoreactive area were significantly reduced in $\mathrm{Mdk}^{-1-}$ mice compared to $\mathrm{Mdk}^{+/+}$mice at 3 days. The CD16/32- and arginase-1-immunoreactive areas were significantly decreased at 7 days (b). RT-qPCR analysis revealed the mRNA levels of the M1 phenotype markers (TNF-a, CD11b) to be significantly downregulated in $\mathrm{Mdk}^{-/-}$than in $\mathrm{Mdk}^{+/+}$mice (c). Data are presented as mean \pm SE ( $n=5$ mice/group in immunohistochemistry, $n=3-4$ mice/group in RT-qPCR). ${ }^{*} p<0.05,{ }^{* *} p<0.01$ (comparison with $\mathrm{MK}^{+/+}$and $\mathrm{Mdk}^{-/-}$). \#\#p $<0.01$ (comparison with 3 days and 7 days). Scale bar $=50 \mu \mathrm{m}$ (all panels)

$\left(\mathrm{CD} 163^{+}\right.$, arginase $\left.-1^{+}\right)$through flow cytometry analysis at 3 days after TBI (Fig. 4). We found the expression of $\mathrm{CD} 4{ }^{+} \mathrm{CD} 25^{+} \mathrm{T}$ cells to be higher in $\mathrm{Mdk}^{-/-}$than in $\mathrm{Mdk}^{+/+}$mice. However, a statistically difference was not observed (Fig. 4a; $p=0.090, t$ test). The expression of the M1 markers, i.e., $\mathrm{CD}^{+} 0^{+}$microglia and $\mathrm{iNOS}^{+}$microglia/ macrophages, were decreased in $\mathrm{Mdk}^{-/-}$than in the $\mathrm{Mdk}^{+/+}$mice. However, a significant difference was not observed. In contrast, the expression of the M2 markers, i.e., $\mathrm{CD} 163^{+}$macrophages and arginase $-1^{+}$microglia, was significantly higher in $\mathrm{Mdk}^{-/-}$compared to $\mathrm{Mdk}^{+/+}$ mice (Fig. 4b, c; $p<0.05, t$ test). Furthermore, arginase$1^{+}$macrophage expression was significantly lower in $\mathrm{Mdk}^{-/-}$than in the $\mathrm{Mdk}^{+/+}$mice (Fig. 4b; $p<0.05, t$ test).

\section{MK-deficiency reduced neuronal apoptotic cell death surrounding lesions after TBI}

Finally, the immunoreactivity of the activated apoptotic marker caspase-3 in the perilesional site after TBI was assessed (Fig. 5). Caspase-3-positive cells were detected in the lesion and the perilesional area at 3 and 7 days post-TBI. However, their expression decreased over time and they were scarcely detected at 14 days after TBI
(Fig. 5a, b). Similarly, their expression was significantly lower in $\mathrm{Mdk}^{-/-}$than in $\mathrm{Mdk}^{+/+}$mice at 7 days following TBI $(p=0.008, t$ test). Immunofluorescence staining showed caspase-3-immuno-positive cells to co-localize with the neuronal marker $(\mathrm{NeuN})$, suggesting neuronal apoptosis surrounding the lesion at 7 days after TBI (Fig. 5c). Therefore, we investigated the ratios of caspase-3- and NeuN-positive neurons surrounding the lesion area in all mice and found the ratios of apoptotic neurons to be significantly lower in $\mathrm{Mdk}^{-/-}$than in $\mathrm{Mdk}^{+/+}$mice at 7 days following TBI (Fig. 5d; $p=0.038$, $t$ test).

\section{Discussion}

This study showed MK-deficiency to both reduce the lesion volume and improve the neurological deficits after TBI through the modulation of both neuroinflammation and neuronal apoptosis. Indeed, this results in a decrease in activation of microglia/macrophages, proinflammatory cytokine, and caspase- 3 activity surrounding the lesion at the early phase after TBI. In addition, MK-deficiency was found to suppress M1 microglia/macrophages phenotype marker and to promote M2 phenotype. MK acts as a proinflammatory cytokine and contributes to 


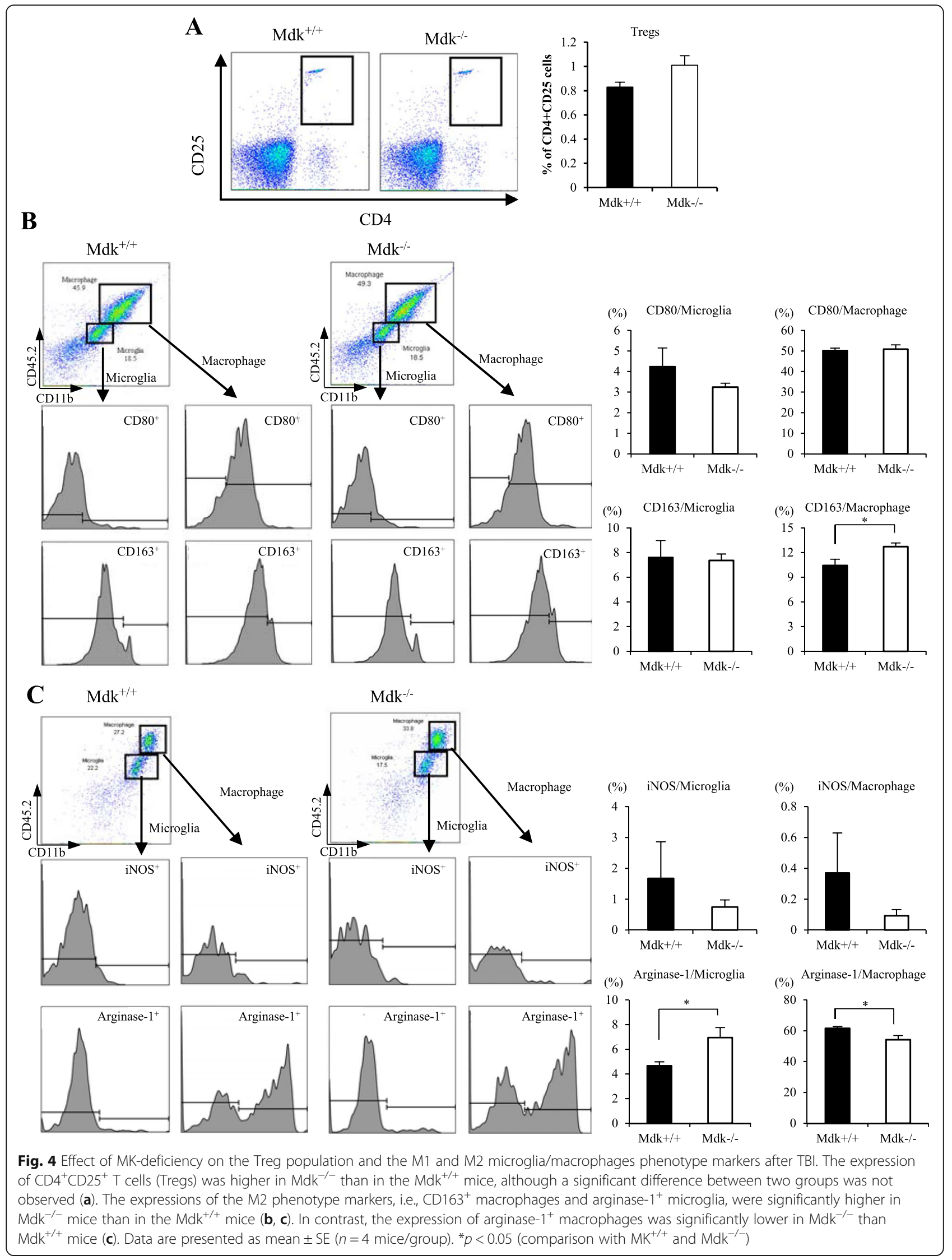




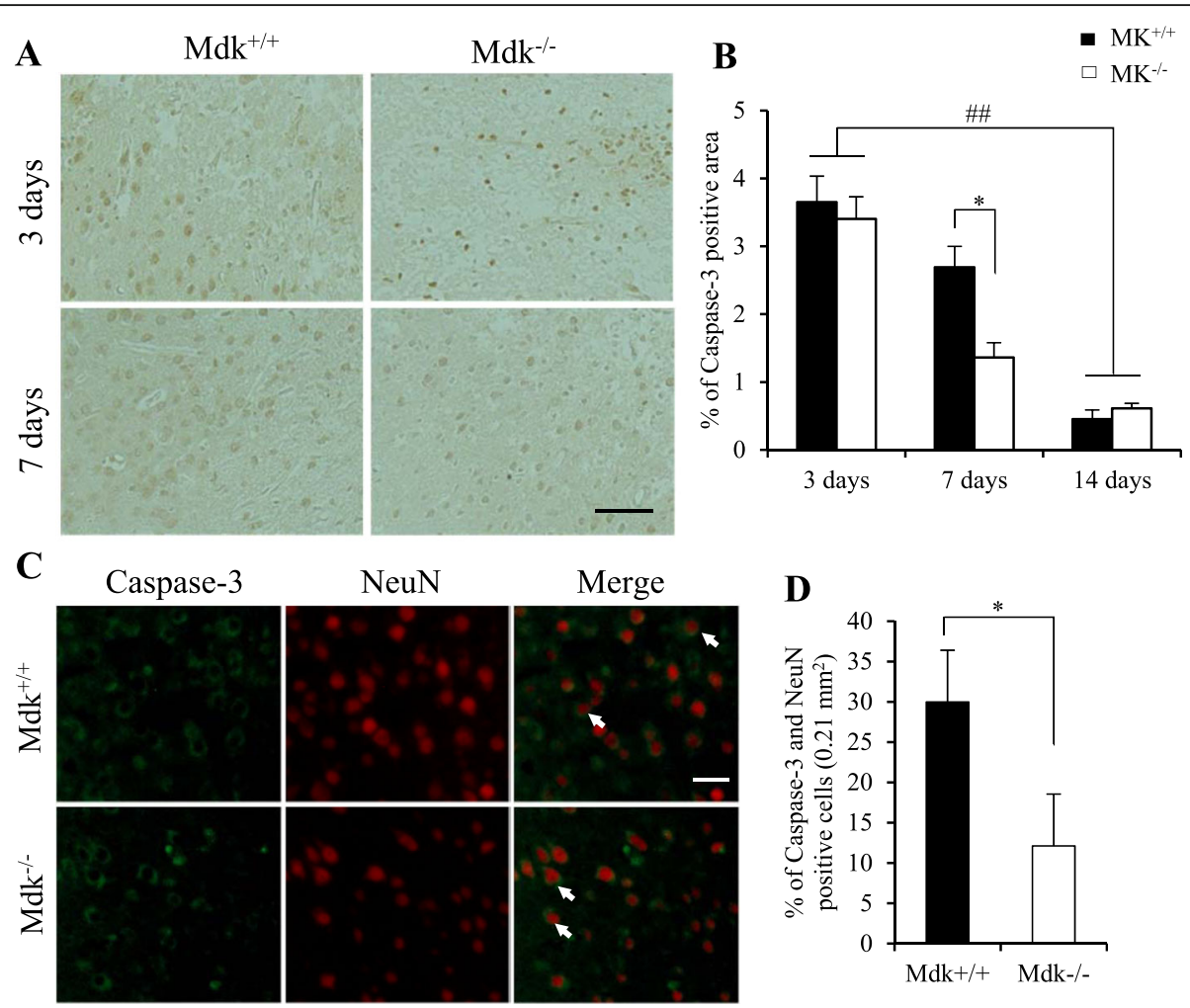

Fig. 5 Effect of MK-deficiency on neuronal apoptosis activity after TBI. Caspase-3-positive cells were detected in the lesion and perilesional areas (a). A higher expression of the caspase-3-positive area was seen in $\mathrm{Mdk}^{-/-}$than in the $\mathrm{Mdk}^{+/+}$mice at 7 days after TBI (b). Moreover, caspase-3immuno-positive cells co-localized with a neuronal marker (NeuN), indicating neuronal apoptosis surrounding the lesion at 7 days after TBI (c). The populations of apoptotic neurons (arrow) were significantly lower in $\mathrm{Mdk}^{-1-}$ than in $\mathrm{Mdk}^{+/+}$mice (d). Data are presented as mean $\pm \mathrm{SE}(n=5$ mice/group). ${ }^{*} p<0.05$ (comparison with $\mathrm{MK}^{+/+}$and $\mathrm{Mdk}^{-/}$). \#\#p $<0.01$ (comparison with 3 days and 14 days). Scale bar $=50 \mu \mathrm{m}$ (all panels of a) and $25 \mu \mathrm{m}$ (all panels of $\mathbf{c}$ )

chronic inflammation through the promotion of chemotaxis and tissue infiltration of neutrophils and microglia/ macrophages [27]. However, MK administration may not directly activate astrocyte or microglia in vitro [35]. Thus, the reduced microglia activation in $\mathrm{Mdk}^{-/-}$mice may have resulted owing to an interference to the migration of neutrophils and macrophages to the injury site at the acute phase after TBI. Wang et al. [26] demonstrated that $\mathrm{Mdk}^{-/-}$EAE mice had fewer infiltrating inflammatory cells in the lumbar spinal cord than $\mathrm{Mdk}^{+/+} \mathrm{EAE}$ mice. In addition, overexpression of the MK gene mediated by p53 remodels the immunosuppressive microenvironment of a glioblastoma by promoting the M2 polarization of microglia [36]. Collectively, our findings suggest the lack of expression of MK gene to reduce both chemotaxis and tissue infiltration of microglia/macrophages, as well as to alter the microglia/macrophages polarization status. This ameliorates neuroinflammation and neuronal apoptosis, consequently leading to reduced tissue loss and favorable neurological outcomes after TBI.

Microglia/macrophages are the primary mediators of the innate response to injury [11]. In fact, a large number of microglia and macrophages proliferate and infiltrate the injury site after TBI [9]. Experimental studies indicate that while neutrophil infiltration in the TBI brain is maximal at 1 day post-injury, it is followed by accumulation of leukocyte subsets that peak at about 3 days post-injury [37]. Inflammatory monocytes are preferentially recruited to brain area subjected to TBI, and they predominate the lesion site at 3 days [8]. In both the spinal cord and the ischemic brain injury, a majority of the microglia and recruited macrophages at the injury site have mixed M1- and M2-like activation profiles [38, 39]. In fact, the mRNA expression of the M1 and M2 phenotype markers rapidly increases after TBI or spinal cord injury [7, 38]. Although the M2 gene expression may fluctuate, the increase in M1 gene expression is sustained over time after TBI [7]. In concordance to previous studies, we also observed a mixed pattern of M1 and M2 phenotype marker expression using immunohistochemistry, RT-qPCR, and flow cytometry. Specially, our results indicated that $\mathrm{Mdk}^{-/-}$mice presented reduced activations of microglia/macrophages in the perilesional site, as well as decreased mRNA levels of M1 phenotype markers (TNF- $\alpha$, CD11b). In addition, they also showed 
increased M2 phenotype marker cells (CD163, arginase1 ) in the acute phase after TBI. While TNF- $\alpha$ is an important glia-mediated proinflammatory cytokine participating in the caspase-3-dependent cell death [40], arginase-1 may be most relevant for CNS repair [38]. Our findings suggest that absence of the MK gene might modulate the M1 and M2 phenotype polarization of microglia/macrophages, thereby alleviating neuroinflammation and neuronal apoptosis after TBI. However, the expression of the M2 phenotype marker was not significantly enhanced, as seen through the immunohistochemical and RT-qPCR analyses. In contrast, the expression of the M1 phenotype marker did not show a significant reduction, as determined by flow cytometry. These discordances may depend on the detection method and the phenotype marker (e.g., surface marker expression and cytokine/chemokine production) used. In addition, the Treg population in TBI brains was not found to be significantly different between $\mathrm{Mdk}^{+/+}$and $\mathrm{Mdk}^{-1-}$ mice. Tregs were reported to both inhibit microglia/macrophages activation and shift their polarization towards the protective M2 phenotype although $\mathrm{CD} 4^{+} \mathrm{CD} 25^{-} \mathrm{T}$ cells displayed the opposite effects by promoting M1 polarization [41-43]. Considering that MK aggravates EAE by decreasing regulatory $\mathrm{CD}^{+}{ }^{+} \mathrm{CD} 25^{+} \mathrm{Foxp}^{+}{ }^{+} \mathrm{T}$ cells (Tregs) [26, 28], further studies should be conducted to clarify the mechanism underlying MK-induced microglia/macrophages polarization after TBI. To the best of our knowledge, this study was the first to suggest that MK could regulate microglia/ macrophages polarization and neuroinflammation following experimental TBI.

MK may act as a modulator of CNS neuroinflammation depending on the inflammatory stimulus [29, 44]. Although our results showed MK-deficiency to regulate microglia-mediated neuroinflammation, the same was not valid for astrocytosis in the perilesional cortex after TBI. While MK reduces the microglia responses and astrocytosis induced by amphetamine in the striatum, LPS-induced neuroinflammation seems to be potentiated by MK [44]. Therefore, TBI-induced glial response may be different from the drug-induced one. In addition, MK-induced microglia response and astrocytosis may present different regulation patterns, based on either the inflammatory stimulus or the observation site (e.g., the cortex or striatum).

In mammals, MK and pleiotrophin (PTN) present an approximately $50 \%$ similarity in their amino acid sequence and comprise a two-member family of heparinbinding cytokines [16]. Both cytokines are novel modulators of neuroinflammation and play different roles based on the inflammatory stimulus and the duration of the neuroinflammatory processes. Therefore, the MK/PTN signaling pathways are novel therapeutic strategies to modulate neuroinflammation in acute and chronic pathological states [44]. Inhibiting MK as well as PTN may be beneficial in neuroinflammatory states that promote exacerbation of neurodegeneration. The potential inhibition of both MK and PTN has been previously studied for the treatment of malignant disease models and EAE mice, including siRNA, shRNA antibody, and RNA aptamers $[26,45,46]$. In contrast, intrathecal administration of MK showed functional recovery after rat model of spinal cord injury [35]. Collectively, MK may be a multifunctional cytokine with a dual therapeutic potential for CNS injury. Therefore, further studies investigating the neuroprotective mechanisms and therapeutic potentials of MK are warranted. In the present study, MK was proposed to be an important factor and a potent regulator of neuroinflammation via the moderation of microglia/macrophages polarization after TBI. Therefore, the inhibiting action of MK may be considered as a potential therapeutic advantage to modulate neuroinflammation when treating TBI.

\section{Conclusions}

The present study demonstrated MK-deficiency to reduce tissue infiltration of microglia/macrophages and alter the M1- and M2-like microglia/macrophages polarization status. These were indicated to result in alleviated neuroinflammation and decreased neuronal apoptosis, leading to reduced tissue loss and improved neurological outcomes after TBI. Therefore, targeting MK to modulate neuroinflammation may be a potential therapeutic strategy for TBI.

\section{Abbreviations}

ANOVA: One-way analysis of variance; CNS: Central nervous system; EAE: Experimental autoimmune encephalomyelitis; FPI: Fluid percussion injury; GFAP: Glial fibrillary acidic protein; HE: Hematoxylin and eosin; Iba1: Ionized calcium-binding adaptor molecule1; IL-10: Interleukin-10; IL-

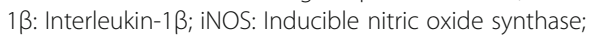
LPS: Lipopolysaccharide; MAPK: Mitogen-activated protein kinase; Mdk $^{-/-}$: MK gene deficient; Mdk $^{+/+}$: Wild type; MK: Midkine; NF-kB: Nuclear factor-kappa B; NSS: Neurological Severity Score; PTN: Pleiotrophin; RT-qPCR: Reverse transcriptase quantitative PCR; TBI: Traumatic brain injury; TNF-a: Tumor necrosis factor-a; Treg: Regulatory $T$ cell

\section{Acknowledgments \\ The authors greatly appreciate the overall advice for study of Dr. Yoshihiro Yoshida and are grateful to Ms. Hui Gao for excellent technical assistance.}

\section{Authors' contributions}

$\mathrm{HS}$ and ST preformed the literature review and wrote the manuscript. ST, SO, $\mathrm{KN}, \mathrm{KN}, \mathrm{AT}, \mathrm{YI}$, and KK performed the experiments. ST carried out the animal study and histochemical and biochemical study. SO, KN, KN, AT, and YI carried out the animal study and quantitative analysis. TM performed the flow cytometry analysis. KK set up the FPI device. All authors read and approved the final manuscript.

\section{Funding}

This work was supported by grant from the JSPS KAKENHI (grant No. JP17K01459 to Harutoshi Sakakima). 


\section{Availability of data and materials}

The datasets used and/or analyzed during the current study are available from the corresponding author on reasonable request.

\section{Ethics approval and consent to participate}

The experimental protocol was approved by the ethics board of the Institute of Experimental Animal Science of Kagoshima University.

\section{Consent for publication}

Not applicable.

\section{Competing interests}

The authors declare that they have no competing interests.

\section{Author details}

'Department of Physical Therapy, School of Health Sciences, Faculty of Medicine, Kagoshima University, 8-35-1, Sakuragaoka, Kagoshima 890-8544, Japan. ${ }^{2}$ Department of Pulmonary Medicine, Graduate School of Medical and Dental Sciences, Kagoshima University, Kagoshima, Japan. ${ }^{3}$ Division of Brain Science, Department of Physiology, Kurume University School of Medicine, Kurume, Japan.

\section{Received: 17 August 2019 Accepted: 12 January 2020}

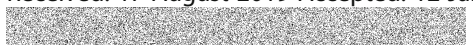

\section{References}

1. Demetriades D, Kimbrell B, Salim A, Velmahos G, Rhee P, Preston C, Gruzinski G, Chan L. Trauma deaths in a mature urban trauma system: is "trimodal" distribution a valid concept? J Am Coll Surg. 2005;201:343-8.

2. Selassie AW, Zaloshnja E, Langlois JA, Miller T, Jones P, Steiner C. Incidence of long-term disability following traumatic brain injury hospitalization, United States, 2003. J Head Trauma Rehabil. 2008;23:123-31.

3. Ghajar J. Traumatic brain injury. Lancet. 2000:356:923-9.

4. Khan M, Sakakima H, Dhammu TS, Shunmugavel A, Im YB, Gilg AG, Singh AK, Singh I. S-nitrosoglutathione reduces oxidative injury and promotes mechanisms of neurorepair following traumatic brain injury in rats. J Neuroinflammation. 2011:8:78.

5. Xu X, Gao W, Cheng S, Yin D, Li F, Wu Y, et al. Anti-inflammatory and immunomodulatory mechanisms of atorvastatin in a murine model of traumatic brain injury. J Neuroinflammation. 2017;14:167.

6. Stoica BA, Faden Al. Cell death mechanisms and modulation in traumatic brain injury. Neurotherapeutics. 2010;7:3-12

7. Wang G, Zhang J, Hu X, Zhang L, Mao L, Jiang X, Liou AK, Leak RK, Gao Y, Chen J. Microglia/macrophage polarization dynamics in white matter after traumatic brain injury. J Cereb Blood Flow Metab. 2013;33:1864-74.

8. Loane DJ, Kumar A. Microglia in the TBI brain: the good, the bad, and the dysregulated. Exp Neurol. 2016;275:316-27.

9. Turtzo LC, Lescher J, Janes L, Dean DD, Budde MD, Frank JA. Macrophagic and microglial responses after focal traumatic brain injury in the female rat. J Neuroinflammation. 2014;11:82.

10. Karve IP, Taylor JM, Crack PJ. The contribution of astrocytes and microglia to traumatic brain injury. Br J Pharmacol. 2016;173:692-702.

11. Loane DJ, Byrnes KR. Role of microglia in neurotrauma. Neurotherapeutics. 2010;7:366-77.

12. Zhou $X$, He $X$, Ren $Y$. Function of microglia and macrophages in secondary damage after spinal cord injury. Neural Regen Res. 2014;9:1787-95.

13. Corrigan F, Mander KA, Leonard AV, Vink R. Neurogenic inflammation after traumatic brain injury and its potentiation of classical inflammation. J Neuroinflammation. 2016:13:264.

14. Tao L, Li D, Liu H, Jiang F, Xu Y, Cao Y, Gao R, Chen G. Neuroprotective effects of metformin on traumatic brain injury in rats associated with NF-KB and MAPK signaling pathway. Brain Res Bull. 2018;140:154-61.

15. Kadomatsu K, Kishida S, Tsubota S. The heparin-binding growth factor midkine: the biological activities and candidate receptors. J Biochem. 2013; 153:511-21.

16. Muramatsu T. Midkine, a heparin-binding cytokine with multiple roles in development, repair and diseases. Proc Jpn Acad Ser B Phys Biol Sci. 2010; 86:410-25

17. Yasuhara O, Muramatsu H, Kim SU, Muramatsu T, Maruta H, McGeer PL. Midkine, a novel neurotrophic factor, is present in senile plaques of Alzheimer disease. Biochem Biophys Res Commun. 1993;192:246-51.
18. Yoshida Y, Goto M, Tsutsui J, Ozawa M, Sato E, Osame M, Muramatsu T. Midkine is present in the early stage of cerebral infarct. Brain Res Dev Brain Res. 1995:85:25-30.

19. Sakakima H, Yoshida Y, Muramatsu T, Yone K, Goto M, ljiri K, et al. Traumatic injury-induced midkine expression in the adult rat spinal cord during the early stage. J Neurotrauma. 2004;21:471-7.

20. Tsutsui J, Kadomatsu K, Matsubara S, Nakagawara A, Hamanoue M, Takao S, et al. A new family of heparin-binding growth/differentiation factors: increased midkine expression in Wilms' tumor and other human carcinomas. Cancer Res. 1993;53:1281-5.

21. Horiba M, Kadomatsu K, Nakamura E, Muramatsu H, Ikematsu S, Sakuma S, et al. Neointima formation in a restenosis model is suppressed in midkinedeficient mice. J Clin Invest. 2000;105:489-95.

22. Sato W, Kadomatsu K, Yuzawa Y, Muramatsu H, Hotta N, Matsuo S, et al. Midkine is involved in neutrophil infiltration into the tubulointerstitium in ischemic renal injury. J Immunol. 2001;167:3463-9.

23. Ochiai K, Muramatsu $H$, Yamamoto $S$, Ando $H$, Muramatsu T. The role of midkine and pleiotrophin in liver regeneration. Liver Int. 2004;24:484-91.

24. Weckbach LT, Gola A, Winkelmann M, Jakob SM, Groesser L, Borgolte J, et al. The cytokine midkine supports neutrophil trafficking during acute inflammation by promoting adhesion via beta2 integrins (CD11/CD18). Blood. 2014;123:1887-96.

25. Maruyama K, Muramatsu H, Ishiguro N, Muramatsu T. Midkine, a heparinbinding growth factor, is fundamentally involved in the pathogenesis of rheumatoid arthritis. Arthritis Rheum. 2004;50:1420-9.

26. Wang J, Takeuchi H, Sonobe $Y$, Jin S, Mizuno T, Miyakawa S, et al. Inhibition of midkine alleviates experimental autoimmune encephalomyelitis through the expansion of regulatory T cell population. Proc Natl Acad Sci U S A. 2008;105:3915-20.

27. Aynacıoğlu AŞ, Bilir A, Tuna MY. Involvement of midkine in autoimmune and autoinflammatory diseases. Mod Rheumatol. 2019;29:567-71.

28. Sonobe Y, Li H, Jin S, Kishida S, Kadomatsu K, Takeuchi H, et al. Midkine inhibits inducible regulatory $T$ cell differentiation by suppressing the development of tolerogenic dendritic cells. J Immunol. 2012;188:2602-11.

29. Vicente-Rodriguez M, Fernandez-Calle R, Gramage E, Perez-Garcia C, Ramos MP, Herradon G. Midkine is a novel regulator of amphetamine-induced striatal gliosis and cognitive impairment: evidence for a stimulus-dependent regulation of neuroinflammation by midkine. Mediat Inflamm. 2016:2016:9894504.

30. Fernández-Calle R, Vicente-Rodríguez M, Gramage E, de la Torre-Ortiz C, Pérez-García C, Ramos MP, Herradón G. Endogenous pleiotrophin and midkine regulate LPS-induced glial responses. Neurosci Lett. 2018;662:213-8.

31. Ikutomo M, Sakakima H, Matsuda F, Yoshida Y. Midkine-deficient mice delayed degeneration and regeneration after skeletal muscle injury. Acta Histochem. 2014;116:319-26.

32. Sakakima H, Yoshida $Y$, Yamazaki $Y$, Matsuda F, Ikutomo M, ljiri $K$, et al. Disruption of the midkine gene (Mdk) delays degeneration and regeneration in injured peripheral nerve. J Neurosci Res. 2009:87:2908-15.

33. Tucker LB, Velosky AG, Fu AH, McCabe JT. Chronic neurobehavioral sex differences in a murine model of repetitive concussive brain injury. Front Neurol. 2019;10:509.

34. Clark W, Gunion-Rinker L, Lessov N, Hazel K. Citicoline treatment for experimental intracerebral hemorrhage in mice. Stroke. 1998;29:2136-40.

35. Muramoto A, Imagama S, Natori T, Wakao N, Ando K, Tauchi R, Hirano K, Shinjo R, Matsumoto T, Ishiguro N, Kadomatsu K. Midkine overcomes neurite outgrowth inhibition of chondroitin sulfate proteoglycan without glial activation and promotes functional recovery after spinal cord injury. Neurosci Lett. 2013;550:150-5.

36. Meng X, Duan C, Pang H, Chen Q, Han B, Zha C, Dinislam M, Wu P, Li Z, Zhao S, Wang R, Lin L, Jiang C, Cai J. DNA damage repair alterations modulate M2 polarization of microglia to remodel the tumor microenvironment via the p53-mediated MDK expression in glioma. EBioMedicine. 2019;41:185-99.

37. Soares HD, Hicks RR, Smith D, McIntosh TK. Inflammatory leukocytic recruitment and diffuse neuronal degeneration are separate pathological processes resulting from traumatic brain injury. J Neurosci. 1995;15:8223-33.

38. Kigerl KA, Gensel JC, Ankeny DP, Alexander JK, Donnelly DJ, Popovich PG. Identification of two distinct macrophage subsets with divergent effects causing either neurotoxicity or regeneration in the injured mouse spinal cord. J Neurosci. 2009:29:13435-44.

39. Hu X, Li P, Guo Y, Wang H, Leak RK, Chen S, Gao Y, Chen J. Microglia/ macrophage polarization dynamics reveal novel mechanism of injury expansion after focal cerebral ischemia. Stroke. 2012;43:3063-70. 
40. Nimmervoll B, White R, Yang JW, An S, Henn C, Sun JJ, Luhmann HJ. LPSinduced microglial secretion of TNFa increases activity-dependent neuronal apoptosis in the neonatal cerebral cortex. Cereb Cortex. 2013;23:1742-55.

41. Liu G, Ma H, Qiu L, Li L, Cao Y, Ma J, Zhao Y. Phenotypic and functional switch of macrophages induced by regulatory CD4+CD25+ T cells in mice. Immunol Cell Biol. 2011;89:130-42.

42. Xie L, Choudhury GR, Winters A, Yang SH, Jin K. Cerebral regulatory T cells restrain microglia/macrophage-mediated inflammatory responses via IL-10 Eur J Immunol. 2015;45:180-91.

43. Zhou K, Zhong Q, Wang YC, Xiong XY, Meng ZY, Zhao T, Zhu WY, Liao MF, Wu LR, Yang YR, Liu J, Duan CM, Li J, Gong QW, Liu L, Yang MH, Xiong A, Wang J, Yang QW. Regulatory T cells ameliorate intracerebral hemorrhageinduced inflammatory injury by modulating microglia/macrophage polarization through the IL-10/GSK3B/PTEN axis. J Cereb Blood Flow Metab. 2017;37:967-79.

44. Herradon G, Ramos-Alvarez MP, Gramage E. Connecting metainflammation and neuroinflammation through the PTN-MK-RPTP $\beta / \zeta$ axis: relevance in therapeutic development. Front Pharmacol. 2019;10:377.

45. Muramatsu T. Midkine: a promising molecule for drug development to treat diseases of the central nervous system. Curr Pharm Des. 2011;17:410-23.

46. Shi Y, Ping YF, Zhou W, He ZC, Chen C, Bian BS, Zhang L, Chen L, Lan X, Zhang XC, Zhou K, Liu Q, Long H, Fu TW, Zhang XN, Cao MF, Huang Z, Fang X, Wang X, Feng H, Yao XH, Yu SC, Cui YH, Zhang X, Rich JN, Bao S, Bian XW. Tumour-associated macrophages secrete pleiotrophin to promote PTPRZ1 signalling in glioblastoma stem cells for tumour growth. Nat Commun. 2017:8:15080.

\section{Publisher's Note}

Springer Nature remains neutral with regard to jurisdictional claims in published maps and institutional affiliations.

Ready to submit your research? Choose BMC and benefit from:

- fast, convenient online submission

- thorough peer review by experienced researchers in your field

- rapid publication on acceptance

- support for research data, including large and complex data types

- gold Open Access which fosters wider collaboration and increased citations

- maximum visibility for your research: over $100 \mathrm{M}$ website views per year

At BMC, research is always in progress.

Learn more biomedcentral.com/submissions 